\title{
Fe-B Nanocapsules Characterized by Analytical Electron Microscopy
}

\author{
J.G Zheng*§ , Z.D. Zhang ${ }^{+}$, V.P. Dravid*
}

*Electron Probe Instrumentation Center (EPIC), NUANCE; Department of Materials Science and Engineering, Northwestern University, Evanston, IL 60208-3108, USA

${ }^{+}$Shenyang National Laboratory for Materials Science and International Center for Materials

Physics, Institute of Metal Research, Academic Sinica, Shengyany 110015, P.R. China

Magnetic metal particles in nanometer scale provide a unique dimensionally confined system, exhibit particle size related properties and have great potential in drug delivery, magnetic fluid and magnetic recording. To protect the fine metal particles from oxidation, magnetic nanocapusules with a core/shell structure have been developed. Most of the nanocapsules were prepared in carbon system, that is, the particles were encapsulated in a carbon shell with graphite crystal structure. Three years ago, we fabricated magnetic nanocapusles in boron system, consisting of various crystalline Fe-B cores and amorphous B-O shells [1]. In order to investigate if the cores are protected by the amorphous B-O shells and understand the formation of crystalline cores, the Fe-B nanocapsules were recently re-examined using high-resolution transmission electron microscopy (HRTEM), energy-filtered transmission electron microscopy (EFTEM), energy dispersive X-ray (EDX) microanalysis and micro-diffraction (MD). A combination of these techniques proves powerful to characterize complex structural features at the nanometer scale.

The well-dispersed nanocapsules were prepared by arc discharge in diborane [1]. Bright field micrograph and EFTEM elemental maps (Fig.1) show the shell/core morphology of the nanocapsules in which oxygen is still only located in the shells.

The crystalline cores still remain unchanged and contain several phases. $\mathrm{Fe}_{23} \mathrm{~B}_{6}$ (Figs. 2 and 3 ) with fcc structure $(\mathrm{a}=1.07 \mathrm{~nm})$ has been newly identified, together with $\alpha-\mathrm{Fe}(\mathrm{B}), \mathrm{FeB}, \mathrm{Fe}_{3} \mathrm{~B}$ and $\gamma$ - $\mathrm{Fe}(\mathrm{B})$. A close relationship between $\mathrm{Fe}_{23} \mathrm{~B}_{6}$ and $\gamma$ - $\mathrm{Fe}(\mathrm{B})$ phases has been found from both diffraction patterns and images, suggesting a possible phase transformation (Fig. 3). The cores can be one of Fe-B phases or a combination of two or more phases. Statistics of micro-diffraction data indicate that the cores containing metastable $\mathrm{Fe}_{3} \mathrm{~B}$ or $\mathrm{Fe}_{23} \mathrm{~B}_{6}$ phases which are normally formed during quenching account for $80 \%$ of the total cores.

An amorphous or partially crystallized iron oxide layer has been identified between the boron oxide shell and the core, and is attributed to poor vacuum in the sample preparation chamber instead of post-oxidation.

These recent results indicate that the shell can well protect the core from oxidation even the shell is amorphous and contains oxygen, and the quenching procedure in sample preparation plays an important role in forming the core phases.

[1] Z.D. Zhang, J.L. Yu, J.G. Zheng, I. Skorvanek, J. Koac, X.L. Dong, Z.J. Li, S.R. Jin, H.C. Yang, Z.J. Guo, W. Liu, X.G. Zhao, Phys. Rev. B., 64 (2001), 244041

${ }^{\S}$ Correspondence: Phone 847-491-7807, Fax: 847-491-7820, e-mail: j-zheng3@northwestern.edu 


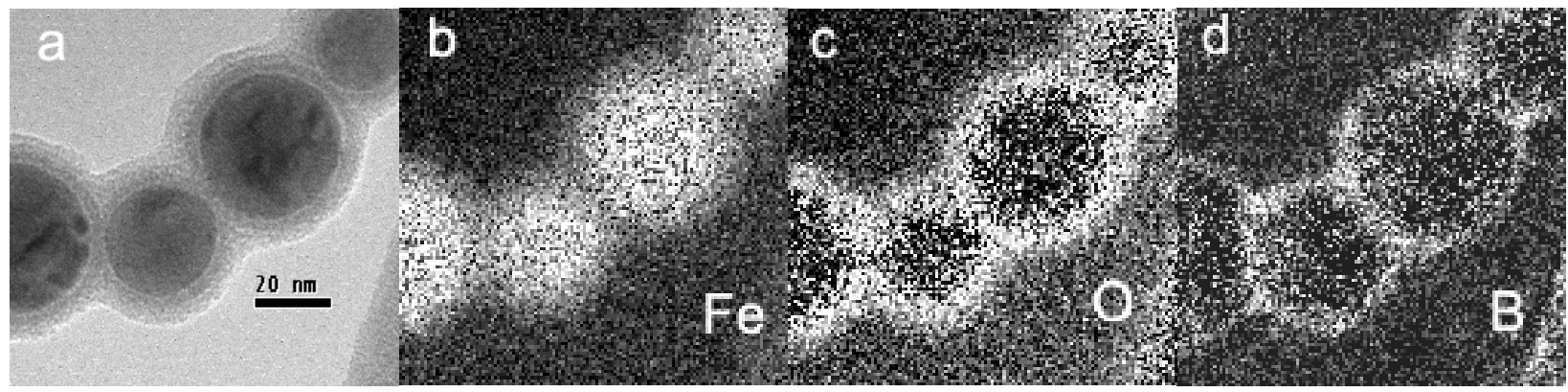

Figure 1 (a) Bright field TEM micrograph of Fe-B nanocapsules. (b)-(d) EFTEM images show elemental maps of $\mathrm{Fe}, \mathrm{O}$ and $\mathrm{B}$, respectively.
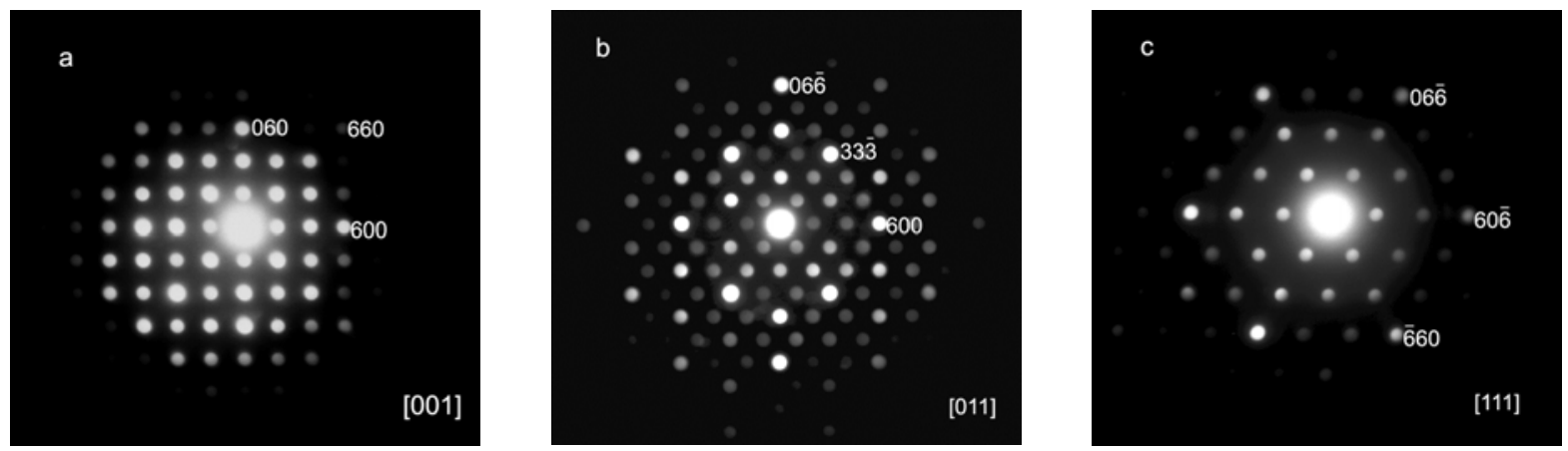

Figure 2. (a)-(c) Micro-diffraction patterns recorded from a $\mathrm{Fe}_{23} \mathrm{~B}_{6}$ core along the [001], [011] and [111] zone axes, respectively.

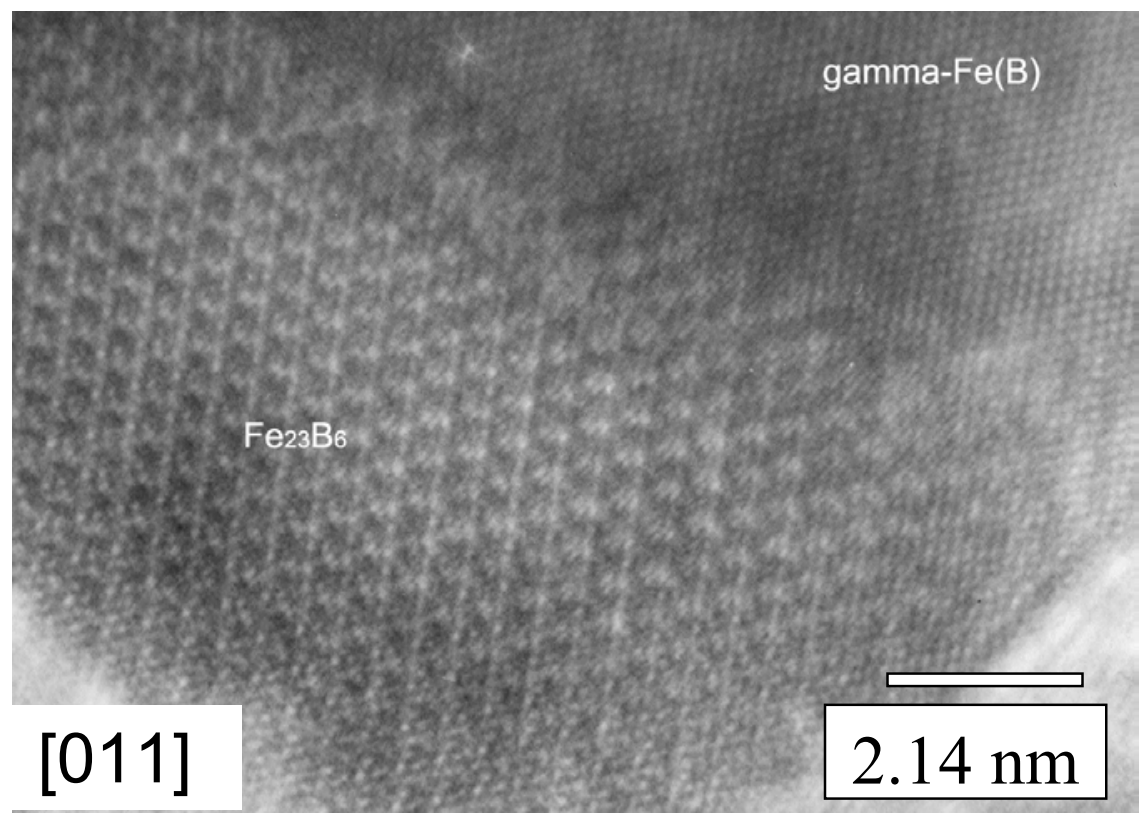

Figure 3. HRTEM image of a core containing $\gamma$ - $\mathrm{Fe}(\mathrm{B})$ and $\mathrm{Fe}_{23} \mathrm{~B}_{6}$ phases recorded along the [011] zone axis. 